\title{
Bielschowskysin, a Gorgonian-Derived Antiplasmodial Diterpene with an Unprecedented Carbon Skeleton
}

Jeffrey Marrero, Abimael D. Rodríguez, ${ }^{*}$ Peter Baran, Raphael G. Raptis, Juan A. Sánchez, Eduardo Ortega-Barria, and Todd L. Capson

Deparment of Chemistry, University of Puerto Rico, P.O. Box 23346, U.P.R. Station, San Juan, Puerto Rico 00931-3346, Deparment of Systematic Biology \& Laboratories of Analytical Biology, Smithsonian Institution, P.O. Box 37012, Washington, D.C. 20013-7012, USA, Florida State University-Panamá, Cell and Molecular Biology of Pathogens Unit, Institute for Tropical Medicine and Health Sciences, La Boca 1034, Balboa, Ancón, and Smithsonian Tropical Research Institute, P.O. Box 2072, Balboa, Ancón, Panamá

\section{List of Supporting Information:}

(1) Detailed description of experimental procedures.

(2) ORTEP drawings of the two symmetry independent molecules of bielschowskysin (1).

(3) Copies of ${ }^{1} \mathrm{H}$ - and ${ }^{13} \mathrm{C}-\mathrm{NMR}$ spectra for bielschowskysin (1).

(4) Tables of crystal data for bielschowskysin (1) (crystal data and structure refinement, atomic coordinates, bond lengths and angles, anisotropic displacement parameters and hydrogen coordinates). 


\section{Experimental Section}

General Experimental Procedures: 1D- and 2D-NMR spectral data were recorded with a Bruker DRX-500 FT-NMR spectrometer. Infrared spectra were obtained with a Nicolet Magna FT-IR 750. The optical rotation was obtained with an Autopol IV automatic polarimeter. Column chromatography was performed on silica gel (35-75 mesh) and TLC analyses were carried out using glass precoated silica gel plates. HPLC was performed using a Polar Bonded Cyano Ultrasphere semipreparative column $(5.0 \mathrm{~mm} \times 25 \mathrm{~cm})$. All solvents used were either spectral grade or were distilled from glass prior to use. The percentage yield of bielschowskysin (1) is based on the weight of the crude $\mathrm{CH}_{2} \mathrm{Cl}_{2} / \mathrm{MeOH}$ extract.

Collection of Pseudopterogorgia kallos: P. kallos (Bielschowsky) was collected by SCUBA at depths of $83-91 \mathrm{ft}$ in Old Providence Island, Colombia, on March 15, 2002. A voucher specimen is stored in the Chemistry Department of the University of Puerto Rico, Río Piedras Campus.

Extraction and Isolation Procedure: The organism was partially airdried, frozen and lyophilized prior to its extraction. The dry organism (1.07 kg) was blended using $\mathrm{CH}_{2} \mathrm{Cl}_{2}-\mathrm{MeOH}(1: 1)(20 \times 1 \mathrm{~L})$. After filtration the crude extract was evaporated and stored under vacuum to yield a green gum (166 g). The crude extract was partitioned between hexane and $\mathrm{H}_{2} \mathrm{O}, \mathrm{CHCl}_{3}$ and $\mathrm{H}_{2} \mathrm{O}$, and then the aqueous suspension was extracted with EtOAc $(2 \times 2 \mathrm{~L})$. The resulting EtOAc extract was concentrated in vacuo to yield $1.47 \mathrm{~g}$ of a green amorphous solid which was chromatographed over Si gel $(60 \mathrm{~g})$ and separated into 10 fractions (I-X) on the basis of TLC analysis. Fraction VII $(347.1 \mathrm{mg})$ was purified by normal-phase HPLC (Ultrasphere Polar-Bonded Cyano column with $25 \%$ IPA in hexane) to afford bielschowskysin (1) $(39.6 \mathrm{mg}$, yield = $0.024 \%$ ) as a colorless crystalline solid. Bielschowskysin (1) was recrystallized by slow evaporation of a mixture of EtOAc/MeOH to yield cubic colorless crystals of excellent quality. 
Bielschowskysin (1): colorless crystalline solid; mp $139-141^{\circ} \mathrm{C},[\alpha]^{20}{ }_{\mathrm{D}}-$ $17.3^{\circ}$ (c 1.1, MeOH); IR (thin film) 3400, 2971, 2932, 2881, 1739, 1658, 1441, 1374, 1250, 1022, 984, $910 \mathrm{~cm}^{-1} ;{ }^{1} \mathrm{H}$ NMR $\left(\mathrm{CD}_{3} \mathrm{OD}, 500 \mathrm{MHz}\right)$ and ${ }^{13} \mathrm{C}$ NMR (CD $\mathrm{OD}, 125 \mathrm{MHz}$ ) (see Table 1); EIMS $\mathrm{m} / \mathrm{z} 374(29), 356(43)$, 346(47), 328(29), 310(18), 287(59), 269(49), 259(40), 241(33), 213(33), 175(32), 153(100), 121(43), 111(82), 101(57), 91(81), 77(75); HREIMS $\mathrm{m} / \mathrm{z}$ [M-AcOH] ${ }^{+} 374.1368$ (calcd for $\mathrm{C}_{20} \mathrm{H}_{22} \mathrm{O}_{7}, 374.1366$ ). Several attempts to detect the expected molecular ion species of 1 were made by LRFABMS. However, due to the rather poor solubility of $\mathbf{1}$ in the two matrices assayed (magic bullet and 3-NBA) no ionic species ascribable to 1 could be detected.

\section{X-ray Single-Crystal Structure Determination of} Bielschowskysin॰0.6 $\mathrm{H}_{\mathbf{2}} \mathrm{O}$ (1) at $\mathbf{2 9 8 ( 2 )} \mathrm{K}$. Crystal data: $\mathrm{C}_{22} \mathrm{H}_{27.2} \mathrm{O}_{9.6}$, $M_{r}=445.24$, monoclinic, space group $P 2_{1}$ (No.4), $a=11.243(2), b=$ 15.640(3), $c=12.376(2) \AA, \beta=94.421(2)^{\circ}, V=2169.6(6) \AA^{3}, Z=4, \rho_{\text {calc }}$ $=1.363 \mathrm{Mgm}^{-3}, F_{000}=944, \lambda\left(\mathrm{Mo}_{\mathrm{K} \alpha}\right)=0.71073 \AA, \mu=0.107 \mathrm{~mm}^{-1}$. Data collection and reduction: crystal size, $0.50 \times 0.39 \times 0.34 \mathrm{~mm}^{3}, \theta$ range,

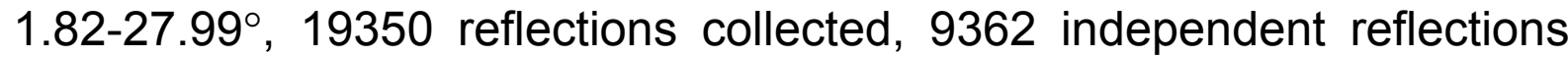
$\left(R_{\text {int }}=0.0291\right)$, final $R$ indices $(I>2 \sigma(I)): R_{1}=0.0372, w R_{2}=0.0951$ for 589 variable parameters, GOF $=1.043$. 


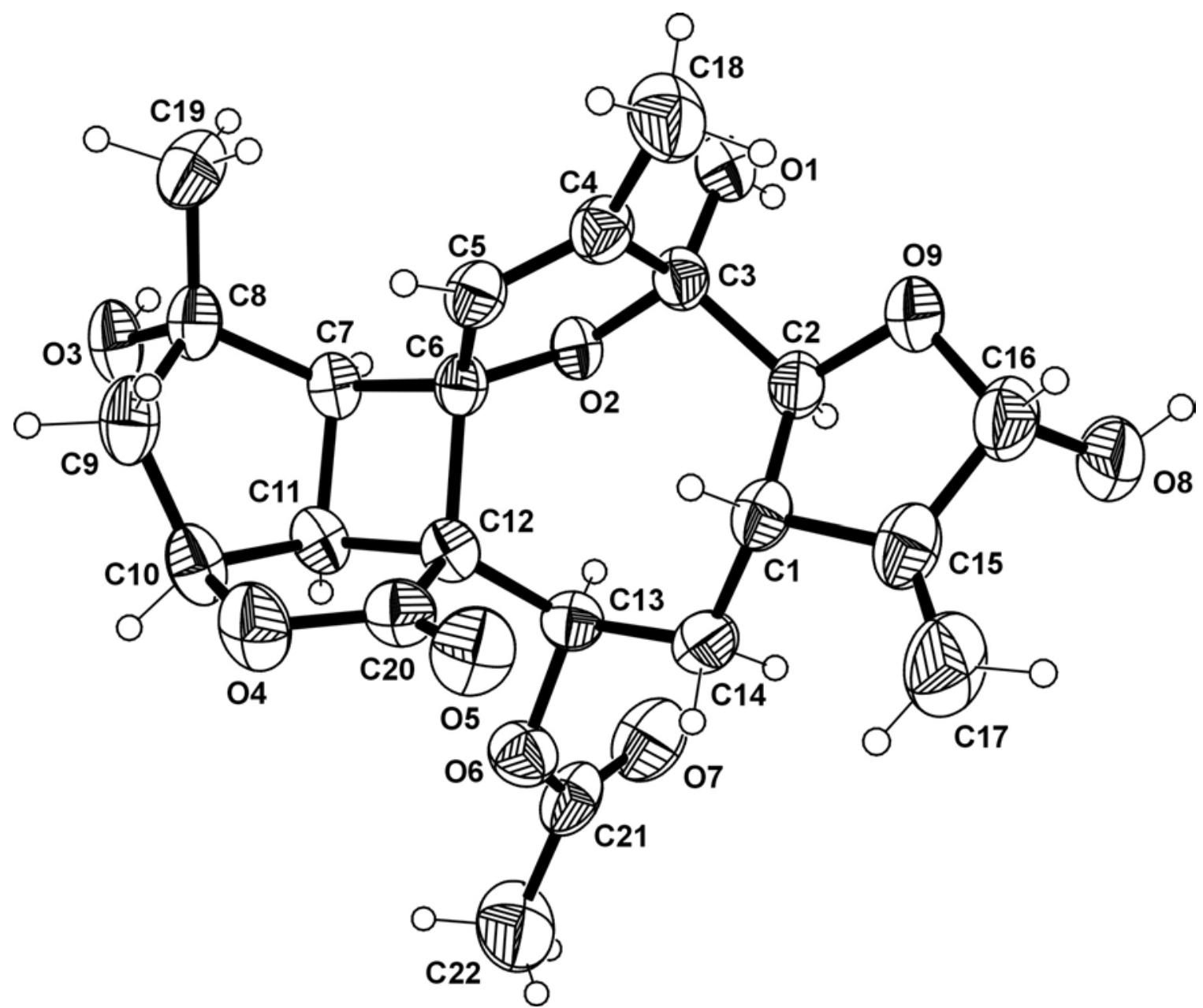

Figure S1. ORTEP diagram of one of the symmetry independent molecules in $\mathbf{1}$. The carbon and oxygen atoms are drawn as 50\% thermal ellipsoids. 


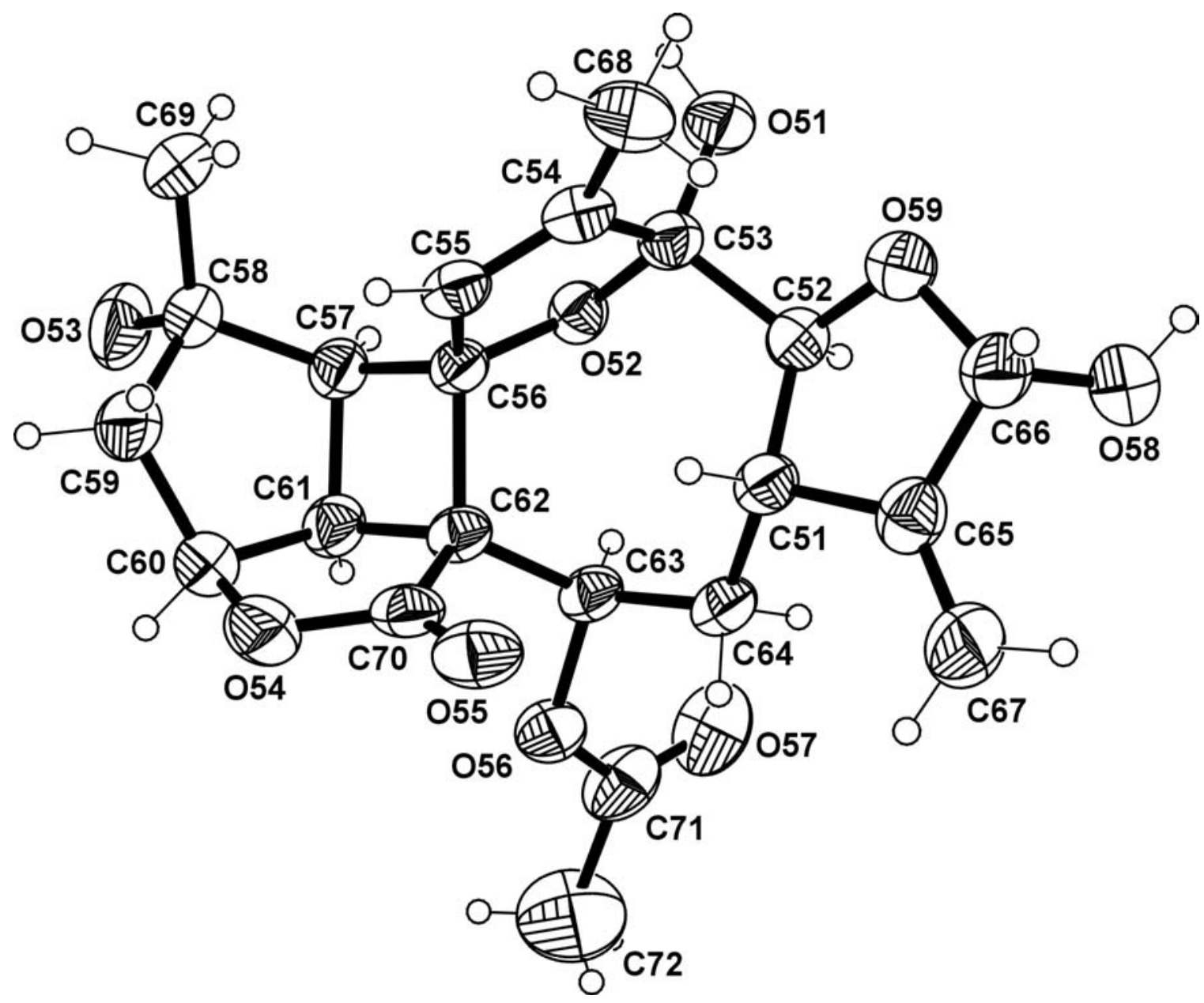

Figure S2. ORTEP diagram of one of the symmetry independent molecules in $\mathbf{1}$. The carbon and oxygen atoms are drawn as 50\% thermal ellipsoids. 


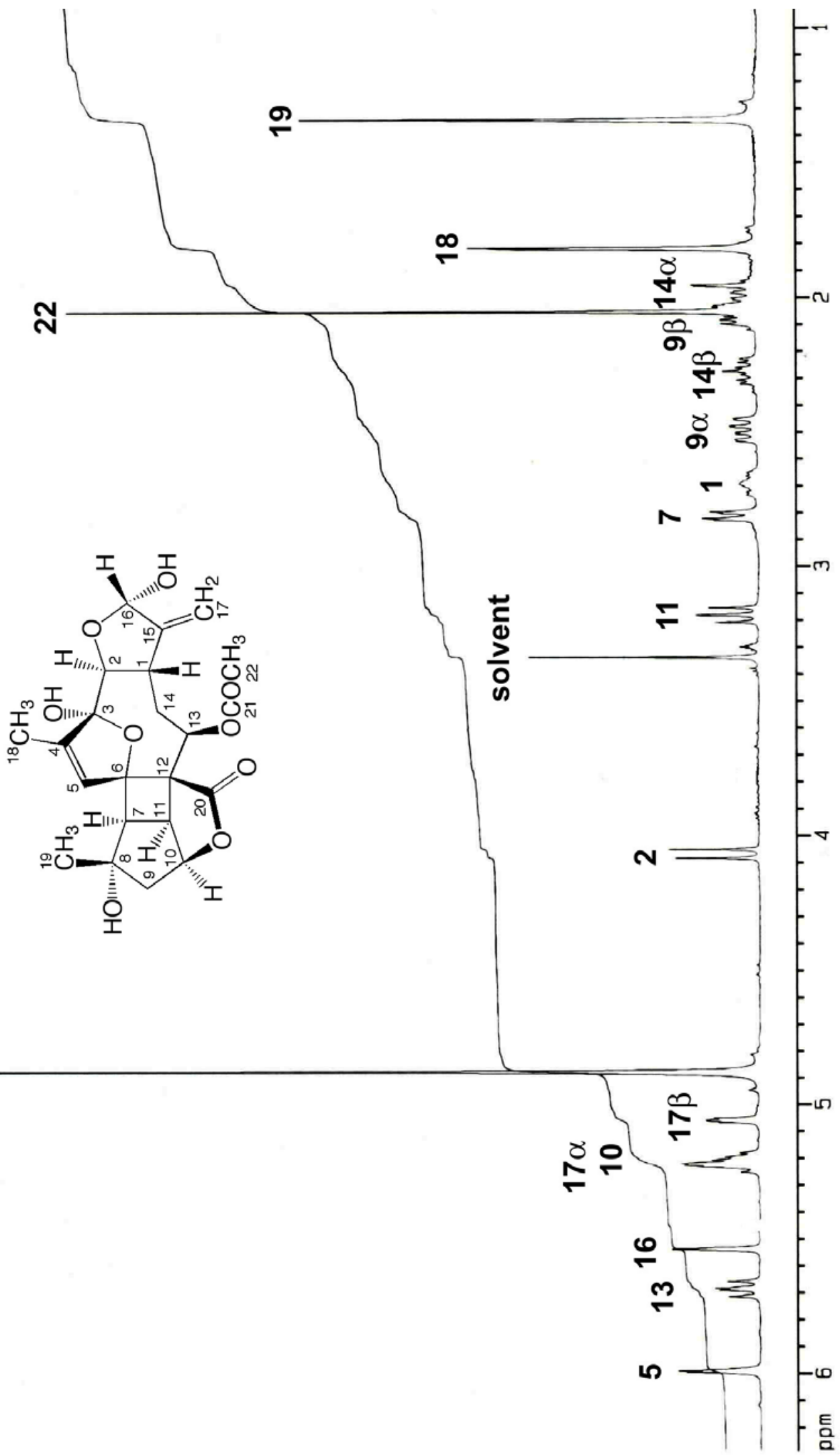




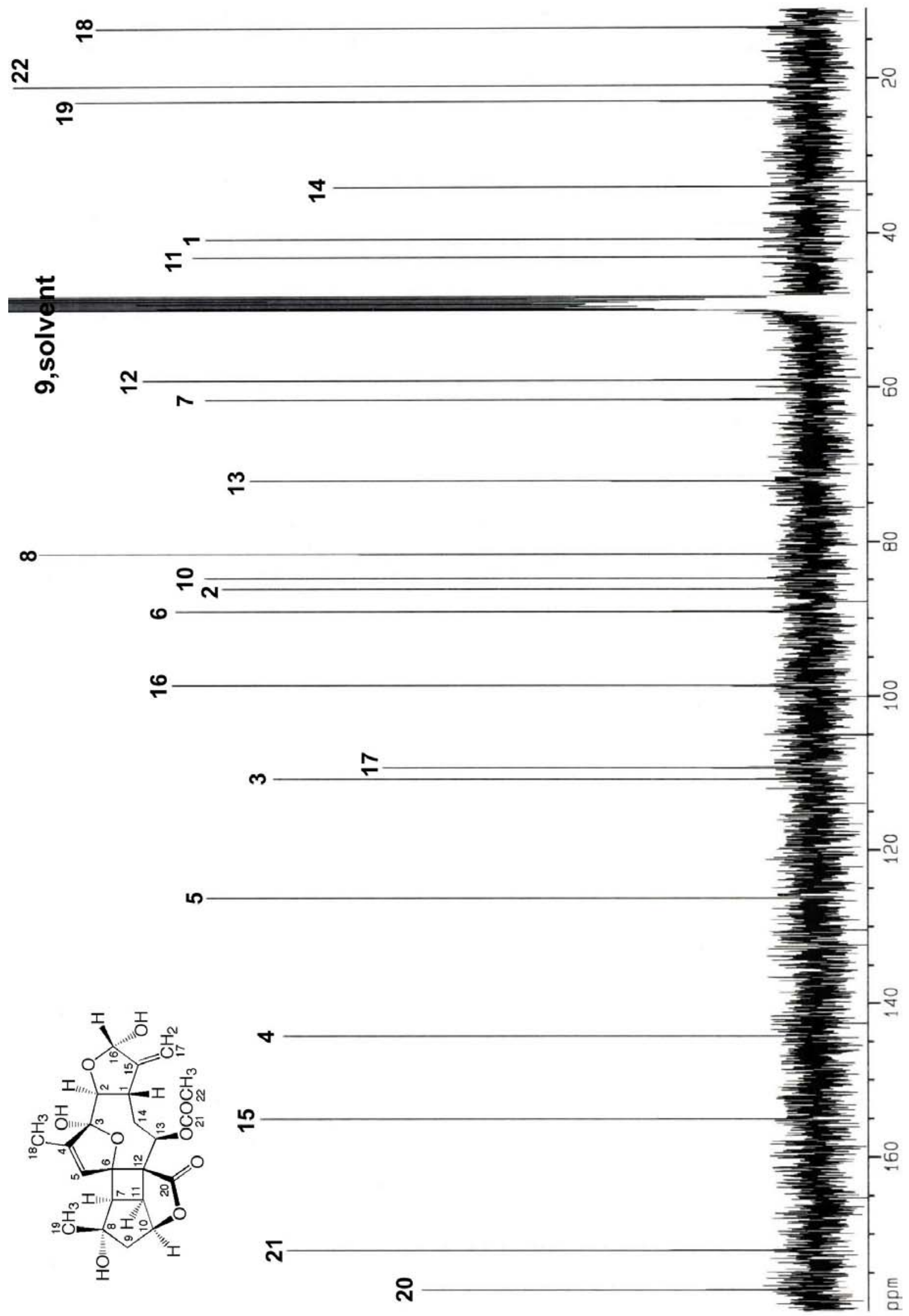


Table 1: Crystal data and structure refinement for Bielschowskysin (1).

\begin{tabular}{|c|c|}
\hline \multicolumn{2}{|c|}{ Bielschowskysin $\left(\mathrm{C}_{22} \mathrm{H}_{26} \mathrm{O}_{9} \_.6 \mathrm{H}_{2} \mathrm{O}\right)$} \\
\hline Empirical formula & $\mathrm{C}_{22} \mathrm{H}_{27.2} \mathrm{O}_{9.6}$ \\
\hline Formula weight & 445.24 \\
\hline Temperature (K) & $298(2)$ \\
\hline Wavelength $(\AA)$ & 0.71073 \\
\hline Crystal system & Monoclinic \\
\hline Space group & $\mathrm{P} 2_{1}($ No.4) \\
\hline \multicolumn{2}{|l|}{ Unit cell dimensions } \\
\hline $\mathrm{a}(\AA)$ & $11.243(2)$ \\
\hline $\mathrm{b}(\AA)$ & $15.640(3)$ \\
\hline c $(\AA)$ & $12.376(2)$ \\
\hline$\alpha(\operatorname{deg})$ & 90 \\
\hline$\beta(\operatorname{deg})$ & $94.421(2)$ \\
\hline$\gamma(\mathrm{deg})$ & 90 \\
\hline Volume $\left(\AA^{3}\right)$ & $2169.6(6)$ \\
\hline $\mathrm{Z}$ & 4 \\
\hline Density (calc.) $\left(\mathrm{Mg} \mathrm{m}^{-3}\right)$ & 1.363 \\
\hline Absorption coefficient $\left(\mathrm{mm}^{-1}\right)$ & 0.107 \\
\hline $\mathrm{F}(000)$ & 944 \\
\hline Crystal size (mm) & $0.50 \times 0.39 \times 0.34$ \\
\hline$\theta$ range for data collection ( $\mathrm{deg}$ ) & 1.82 to 27.99 \\
\hline Index ranges & $-14 \leq h \leq 14,-20 \leq k \leq 20,-16 \leq l \leq 15$ \\
\hline Reflections collected & 19350 \\
\hline Independent reflections & $9362[R($ int $)=0.0291]$ \\
\hline Independent reflections $[I>2 \sigma(I)]$ & $8656[R($ int $)=0.0253]$ \\
\hline Max. and min. transmission & $0.948 / 0.880$ \\
\hline Refinement method & Full-matrix least-squares on $F^{2}$ \\
\hline
\end{tabular}


Data/restraints/parameters

Goodness-of-fit on $\mathrm{F}^{2}$

Final $R$ indices $[I>2 \sigma(I)]$

$R$ indices (all data)

Largest diff. peak and hole (e $\left.\AA^{-3}\right)$
$9362 / 1 / 589$

1.043

$R 1=0.0372, w R 2=0.0951$

$R 1=0.0411, w R 2=0.0981$

0.235 and -0.175
$\& L 120$

$\& F$

\&W64

\&! Table 1. Atomic coordinates [ $\left.\mathrm{x} 10 \&^{\wedge} 4 \& 0\right]$ and equivalent isotropic displacement parameters $[\& \mathrm{GA} \& \wedge \& 0 \times 10 \& \wedge 3 \& 0]$ for ar035. U(eq) is defined as

one third of the trace of the orthogonalized \&IU\&vij\&O\&:\&! tensor.\&N $\& \mathrm{C}$

$\& \mathrm{E}$

$\& \mathrm{~J} 2$

$\& \mathrm{C}$

$\begin{array}{llll}\mathrm{y} & \mathrm{z} & \mathrm{U}(\mathrm{eq})\end{array}$

$\& \mathrm{E}$

$\& \mathrm{C}$

$O(1)$

$O(2)$

O (3)

$O(4)$

O (5)

O (6)

$O(7)$

O (8)

$O(9)$

C (1)

C (2)

C (3)

C (4)

C (5)

C (6)

C (7)

C (8)

C (9)

C (10)

C (11)

C (12)

C (13)

C (14)

C (15)

C (16)

C (17)

C (18)

C (19)

C (20)

C (21)
$8677(1)$

7997 (1)

7751 (1)

4753 (1)

4377 (1)

$6773(1)$

8507 (2)

$7318(2)$

$7220(2)$

$6325(2)$

7435 (2)

7705 (2)

6649 (2)

$6263(2)$

$7016(1)$

7509 (1)

7055 (2)

5795 (2)

$5759(2)$

$6889(1)$

6451 (1)

$6995(2)$

6507 (2)

$5866(2)$

$6569(2)$

4989 (2)

$6189(2)$

$7141(2)$

$5101(2)$

7615 (2)

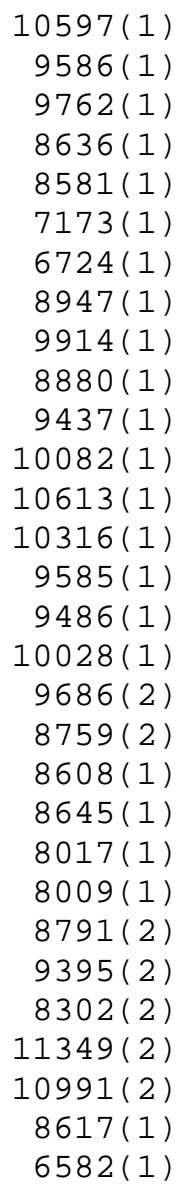

$\begin{aligned} 11021(1) & 45(1) \\ 9767(1) & 33(1) \\ 5948(1) & 49(1) \\ 7591(1) & 55(1) \\ 9324(1) & 56(1) \\ 9188(1) & 47(1) \\ 9995(2) & 65(1) \\ 13971(1) & 70(1) \\ 12541(1) & 57(1) \\ 11334(1) & 39(1) \\ 11577(1) & 39(1) \\ 10699(1) & 36(1) \\ 10253(2) & 40(1) \\ 9291(1) & 36(1) \\ 8960(1) & 32(1) \\ 7817(1) & 34(1) \\ 6831(1) & 42(1) \\ 6535(2) & 49(1) \\ 6923(2) & 46(1) \\ 7681(1) & 37(1) \\ 8832(1) & 34(1) \\ 9668(2) & 37(1) \\ 10793(2) & 44(1) \\ 12454(2) & 49(1) \\ 13216(2) & 56(1) \\ 12730(2) & 75(1) \\ 10876(2) & 61(1) \\ 6956(2) & 54(1) \\ 8655(2) & 41(1) \\ 9410(2) & 50(1) \\ & \end{aligned}$




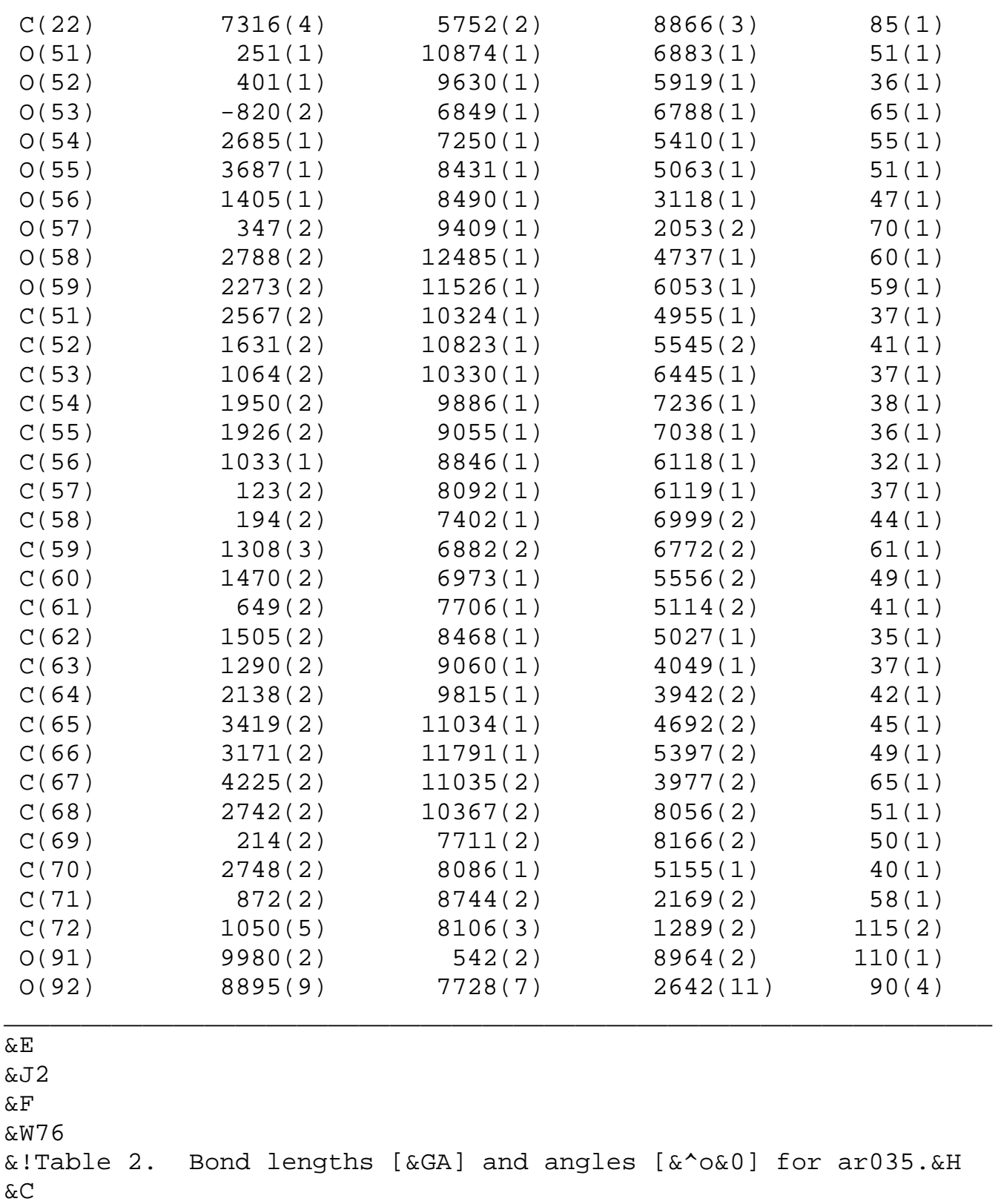

\begin{tabular}{ll}
\hline$\overline{\varepsilon E}$ & \\
$\& D$ & \\
$O(1)-C(3)$ & $1.391(2)$ \\
$O(2)-C(6)$ & $1.4300(19)$ \\
$O(2)-C(3)$ & $1.448(2)$ \\
$O(3)-C(8)$ & $1.454(2)$ \\
$O(4)-C(20)$ & $1.345(2)$ \\
$O(4)-C(10)$ & $1.464(2)$ \\
$O(5)-C(20)$ & $1.205(2)$ \\
$O(6)-C(21)$ & $1.337(3)$ \\
$O(6)-C(13)$ & $1.461(2)$ \\
$O(7)-C(21)$ & $1.210(3)$ \\
$O(8)-C(16)$ & $1.397(3)$ \\
$O(9)-C(16)$ & $1.410(3)$
\end{tabular}




\begin{tabular}{|c|c|}
\hline $\mathrm{O}(9)-\mathrm{C}(2)$ & $1.443(2)$ \\
\hline$C(1)-C(15)$ & $1.522(2)$ \\
\hline$C(1)-C(2)$ & $1.533(3)$ \\
\hline$C(1)-C(14)$ & $1.539(3)$ \\
\hline$C(2)-C(3)$ & $1.529(2)$ \\
\hline$C(3)-C(4)$ & $1.518(3)$ \\
\hline$C(4)-C(5)$ & $1.321(3)$ \\
\hline$C(4)-C(18)$ & $1.500(3)$ \\
\hline$C(5)-C(6)$ & $1.498(2)$ \\
\hline$C(6)-C(7)$ & $1.566(2)$ \\
\hline$C(6)-C(12)$ & $1.604(2)$ \\
\hline$C(7)-C(8)$ & $1.540(2)$ \\
\hline$C(7)-C(11)$ & $1.543(3)$ \\
\hline$C(8)-C(19)$ & $1.516(3)$ \\
\hline$C(8)-C(9)$ & $1.533(3)$ \\
\hline$C(9)-C(10)$ & $1.528(3)$ \\
\hline$C(10)-C(11)$ & $1.538(2)$ \\
\hline$C(11)-C(12)$ & $1.544(2)$ \\
\hline$C(12)-C(20)$ & $1.518(2)$ \\
\hline$C(12)-C(13)$ & $1.521(2)$ \\
\hline$C(13)-C(14)$ & $1.535(3)$ \\
\hline$C(15)-C(17)$ & $1.313(4)$ \\
\hline$C(15)-C(16)$ & $1.514(3)$ \\
\hline$C(21)-C(22)$ & $1.489(4)$ \\
\hline $\mathrm{O}(51)-\mathrm{C}(53)$ & $1.390(2)$ \\
\hline $\mathrm{O}(52)-\mathrm{C}(56)$ & $1.429(2)$ \\
\hline$O(52)-C(53)$ & $1.450(2)$ \\
\hline $\mathrm{O}(53)-\mathrm{C}(58)$ & $1.439(3)$ \\
\hline$O(54)-C(70)$ & $1.347(3)$ \\
\hline $\mathrm{O}(54)-\mathrm{C}(60)$ & $1.457(3)$ \\
\hline $\mathrm{O}(55)-\mathrm{C}(70)$ & $1.199(3)$ \\
\hline$O(56)-C(71)$ & $1.336(3)$ \\
\hline$O(56)-C(63)$ & $1.470(2)$ \\
\hline$O(57)-C(71)$ & $1.199(3)$ \\
\hline$O(58)-C(66)$ & $1.406(3)$ \\
\hline $\mathrm{O}(59)-\mathrm{C}(66)$ & $1.406(2)$ \\
\hline $\mathrm{O}(59)-\mathrm{C}(52)$ & $1.433(2)$ \\
\hline$C(51)-C(65)$ & $1.517(3)$ \\
\hline$C(51)-C(64)$ & $1.532(3)$ \\
\hline$C(51)-C(52)$ & $1.538(2)$ \\
\hline$C(52)-C(53)$ & $1.533(2)$ \\
\hline$C(53)-C(54)$ & $1.510(3)$ \\
\hline$C(54)-C(55)$ & $1.322(3)$ \\
\hline$C(54)-C(68)$ & $1.500(3)$ \\
\hline$C(55)-C(56)$ & $1.494(2)$ \\
\hline$C(56)-C(57)$ & $1.562(3)$ \\
\hline$C(56)-C(62)$ & $1.602(2)$ \\
\hline$C(57)-C(58)$ & $1.531(3)$ \\
\hline$C(57)-C(61)$ & $1.542(2)$ \\
\hline$C(58)-C(69)$ & $1.521(3)$ \\
\hline$C(58)-C(59)$ & $1.538(3)$ \\
\hline$C(59)-C(60)$ & $1.536(3)$ \\
\hline$C(60)-C(61)$ & $1.545(3)$ \\
\hline$C(61)-C(62)$ & $1.541(3)$ \\
\hline$C(62)-C(70)$ & $1.517(2)$ \\
\hline$C(62)-C(63)$ & $1.528(2)$ \\
\hline$C(63)-C(64)$ & $1.529(3)$ \\
\hline
\end{tabular}




\begin{tabular}{|c|c|}
\hline & \\
\hline$C(65)-C(67)$ & $1.315(3)$ \\
\hline$C(65)-C(66)$ & $1.510(3)$ \\
\hline$C(71)-C(72)$ & $1.502(4)$ \\
\hline$\& E$ & \\
\hline$\& D$ & \\
\hline$C(6)-O(2)-C(3)$ & $110.28(12)$ \\
\hline$C(20)-O(4)-C(10)$ & $112.16(14)$ \\
\hline$C(21)-0(6)-C(13)$ & $116.44(16)$ \\
\hline$C(16)-O(9)-C(2)$ & $108.71(16)$ \\
\hline$C(15)-C(1)-C(2)$ & $101.65(16)$ \\
\hline$C(15)-C(1)-C(14)$ & $112.38(16)$ \\
\hline$C(2)-C(1)-C(14)$ & $117.04(15)$ \\
\hline$O(9)-C(2)-C(3)$ & $107.59(15)$ \\
\hline $\mathrm{O}(9)-\mathrm{C}(2)-\mathrm{C}(1)$ & $105.64(15)$ \\
\hline$C(3)-C(2)-C(1)$ & $115.98(15)$ \\
\hline$O(1)-C(3)-O(2)$ & $108.47(14)$ \\
\hline$O(1)-C(3)-C(4)$ & $111.21(15)$ \\
\hline$O(2)-C(3)-C(4)$ & $102.91(13)$ \\
\hline$O(1)-C(3)-C(2)$ & $111.85(14)$ \\
\hline$O(2)-C(3)-C(2)$ & $106.43(14)$ \\
\hline$C(4)-C(3)-C(2)$ & $115.26(15)$ \\
\hline$C(5)-C(4)-C(18)$ & $129.16(18)$ \\
\hline$C(5)-C(4)-C(3)$ & $109.15(15)$ \\
\hline$C(18)-C(4)-C(3)$ & $121.69(17)$ \\
\hline$C(4)-C(5)-C(6)$ & $111.19(15)$ \\
\hline $\mathrm{O}(2)-\mathrm{C}(6)-\mathrm{C}(5)$ & $103.31(13)$ \\
\hline $\mathrm{O}(2)-\mathrm{C}(6)-\mathrm{C}(7)$ & $108.87(12)$ \\
\hline$C(5)-C(6)-C(7)$ & $124.66(14)$ \\
\hline$O(2)-C(6)-C(12)$ & $110.41(13)$ \\
\hline$C(5)-C(6)-C(12)$ & $119.86(13)$ \\
\hline$C(7)-C(6)-C(12)$ & $89.17(13)$ \\
\hline$C(8)-C(7)-C(11)$ & $106.52(14)$ \\
\hline$C(8)-C(7)-C(6)$ & $122.87(14)$ \\
\hline$C(11)-C(7)-C(6)$ & $89.92(13)$ \\
\hline$O(3)-C(8)-C(19)$ & $109.12(17)$ \\
\hline $\mathrm{O}(3)-\mathrm{C}(8)-\mathrm{C}(9)$ & $105.12(15)$ \\
\hline$C(19)-C(8)-C(9)$ & $114.96(18)$ \\
\hline$O(3)-C(8)-C(7)$ & $105.88(15)$ \\
\hline$C(19)-C(8)-C(7)$ & $116.86(17)$ \\
\hline$C(9)-C(8)-C(7)$ & $103.93(15)$ \\
\hline$C(10)-C(9)-C(8)$ & $107.60(15)$ \\
\hline$O(4)-C(10)-C(9)$ & $110.01(17)$ \\
\hline$O(4)-C(10)-C(11)$ & $105.83(14)$ \\
\hline$C(9)-C(10)-C(11)$ & $107.29(16)$ \\
\hline$C(10)-C(11)-C(7)$ & $105.92(16)$ \\
\hline$C(10)-C(11)-C(12)$ & $104.46(13)$ \\
\hline$C(7)-C(11)-C(12)$ & $92.23(13)$ \\
\hline$C(20)-C(12)-C(13)$ & $115.14(15)$ \\
\hline$C(20)-C(12)-C(11)$ & $104.63(14)$ \\
\hline$C(13)-C(12)-C(11)$ & $117.60(14)$ \\
\hline$C(20)-C(12)-C(6)$ & $115.14(14)$ \\
\hline$C(13)-C(12)-C(6)$ & $112.92(13)$ \\
\hline$C(11)-C(12)-C(6)$ & $88.48(13)$ \\
\hline$O(6)-C(13)-C(12)$ & $105.07(14)$ \\
\hline$O(6)-C(13)-C(14)$ & $107.43(15)$ \\
\hline$C(12)-C(13)-C(14)$ & $117.98(15)$ \\
\hline$C(13)-C(14)-C(1)$ & $117.14(16)$ \\
\hline
\end{tabular}




$\begin{array}{lc}C(17)-C(15)-C(16) & 124.6(2) \\ C(17)-C(15)-C(1) & 127.2(2) \\ C(16)-C(15)-C(1) & 108.21(18) \\ O(8)-C(16)-O(9) & 111.8(2) \\ O(8)-C(16)-C(15) & 111.29(19) \\ O(9)-C(16)-C(15) & 105.05(16) \\ O(5)-C(20)-O(4) & 120.81(16) \\ O(5)-C(20)-C(12) & 128.55(18) \\ O(4)-C(20)-C(12) & 110.64(15) \\ O(7)-C(21)-O(6) & 122.5(2) \\ O(7)-C(21)-C(22) & 125.3(2) \\ O(6)-C(21)-C(22) & 112.2(2) \\ C(56)-O(52)-C(53) & 109.74(12) \\ C(70)-O(54)-C(60) & 112.62(15) \\ C(71)-O(56)-C(63) & 116.63(17) \\ C(66)-O(59)-C(52) & 109.45(15) \\ C(65)-C(51)-C(64) & 111.68(15) \\ C(65)-C(51)-C(52) & 101.39(15) \\ C(64)-C(51)-C(52) & 117.93(15) \\ O(59)-C(52)-C(53) & 106.88(15) \\ O(59)-C(52)-C(51) & 104.91(15) \\ C(53)-C(52)-C(51) & 115.35(15) \\ O(51)-C(53)-O(52) & 107.80(14) \\ O(51)-C(53)-C(54) & 116.67(15) \\ O(52)-C(53)-C(54) & 103.61(14) \\ O(51)-C(53)-C(52) & 107.39(15) \\ O(52)-C(53)-C(52) & 106.35(14) \\ C(54)-C(53)-C(52) & 114.32(15) \\ C(55)-C(54)-C(68) & 128.33(19) \\ C(55)-C(54)-C(53) & 109.18(16) \\ C(68)-C(54)-C(53) & 122.43(18) \\ C(54)-C(55)-C(56) & 111.01(16) \\ O(52)-C(56)-C(55) & 103.88(14) \\ O(52)-C(56)-C(57) & 109.30(13) \\ C(55)-C(56)-C(57) & 124.49(14) \\ O(52)-C(56)-C(62) & 111.35(13) \\ C(55)-C(56)-C(62) & 118.45(13) \\ C(57)-C(56)-C(62) & 88.92(13) \\ C(58)-C(57)-C(61) & 107.20(15) \\ C(58)-C(57)-C(56) & 122.29(15) \\ C(61)-C(57)-C(56) & 90.17(13) \\ O(53)-C(58)-C(69) & 108.48(16) \\ O(53)-C(58)-C(57) & 107.29(16) \\ C(69)-C(58)-C(57) & 116.63(17) \\ O(53)-C(58)-C(59) & 107.1(2) \\ C(69)-C(58)-C(59) & 112.88(19) \\ C(57)-C(58)-C(59) & 103.96(15) \\ C(60)-C(59)-C(58) & 106.89(17) \\ O(54)-C(60)-C(59) & 109.4(2) \\ O(54)-C(60)-C(61) & 105.94(16) \\ C(59)-C(60)-C(61) & 107.65(16) \\ C(62)-C(61)-C(57) & 117.53(14) \\ C(62)-C(61)-C(60) & \\ C(57)-C(61)-C(60) & \\ C(70)-C(62)-C(63) & \end{array}$




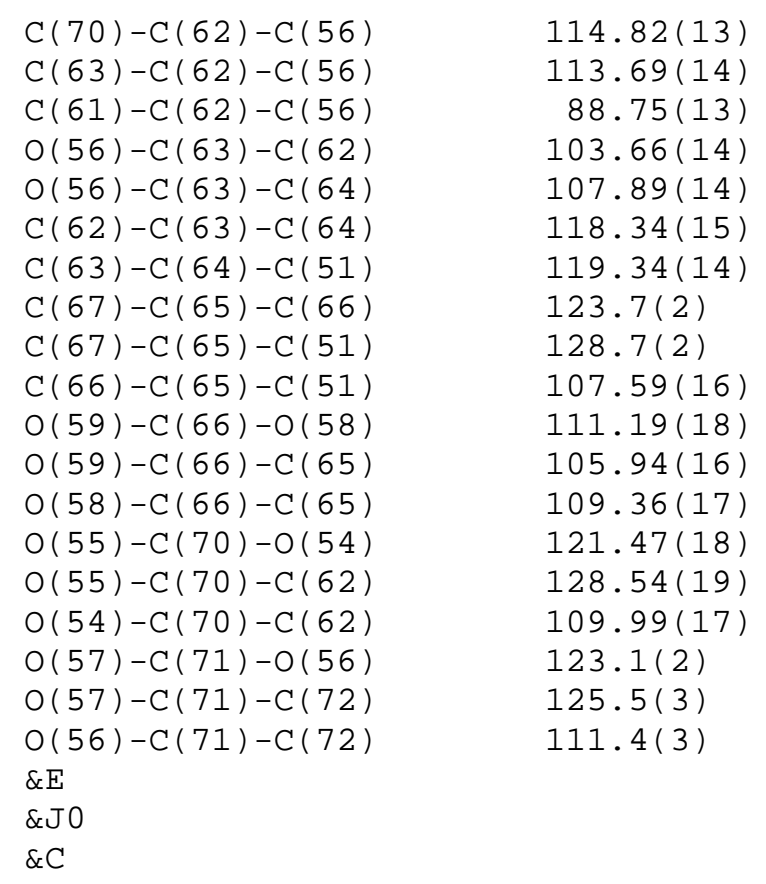

$\begin{array}{ll}48(1) & 37(1) \\ 39(1) & 28(1) \\ 80(1) & 29(1) \\ 83(1) & 50(1) \\ 74(1) & 61(1) \\ 39(1) & 50(1) \\ 64(1) & 70(1) \\ 60(1) & 43(1) \\ 53(1) & 30(1) \\ 44(1) & 34(1) \\ 41(1) & 29(1) \\ 38(1) & 30(1) \\ 39(1) & 36(1)\end{array}$

$-2(1)$
$-1(1)$
$-2(1)$
$2(1)$
$6(1)$
$-5(1)$
$18(1)$
$7(1)$
$-3(1)$
$5(1)$
$2(1)$
$-2(1)$
$3(1)$

$-5(1)$
$-1(1)$
$3(1)$
$-3(1)$
$10(1)$
$0(1)$
$7(1)$
$-3(1)$
$11(1)$
$8(1)$
$2(1)$
$3(1)$
$6(1)$




\begin{tabular}{|c|c|c|c|c|c|c|}
\hline$C(5)$ & $35(1)$ & $38(1)$ & $36(1)$ & $4(1)$ & $3(1)$ & $5(1)$ \\
\hline$C(6)$ & $29(1)$ & $38(1)$ & $29(1)$ & $2(1)$ & $0(1)$ & $2(1)$ \\
\hline C (7) & $29(1)$ & $46(1)$ & $29(1)$ & $-2(1)$ & $2(1)$ & $3(1)$ \\
\hline$C(8)$ & $41(1)$ & $56(1)$ & $29(1)$ & $2(1)$ & $4(1)$ & $5(1)$ \\
\hline$C(9)$ & $35(1)$ & $73(1)$ & $38(1)$ & $7(1)$ & $-2(1)$ & $10(1)$ \\
\hline$C(10)$ & $34(1)$ & $67(1)$ & $35(1)$ & $-8(1)$ & $-1(1)$ & $1(1)$ \\
\hline C (11) & $31(1)$ & $45(1)$ & 35 (1) & $-7(1)$ & $2(1)$ & $3(1)$ \\
\hline$C(12)$ & $29(1)$ & $38(1)$ & $35(1)$ & $-3(1)$ & $2(1)$ & $2(1)$ \\
\hline$C(13)$ & $35(1)$ & $35(1)$ & $40(1)$ & $-1(1)$ & $3(1)$ & $1(1)$ \\
\hline$C(14)$ & $51(1)$ & $39(1)$ & $43(1)$ & $4(1)$ & $8(1)$ & $-3(1)$ \\
\hline$C(15)$ & $51(1)$ & $59(1)$ & $41(1)$ & $7(1)$ & $14(1)$ & $9(1)$ \\
\hline$C(16)$ & $77(1)$ & $56(1)$ & $38(1)$ & $2(1)$ & $14(1)$ & $10(1)$ \\
\hline$C(17)$ & $67(2)$ & $106(2)$ & $56(1)$ & $3(1)$ & $29(1)$ & $-12(2)$ \\
\hline$C(18)$ & $77(2)$ & $57(1)$ & $50(1)$ & $-10(1)$ & $4(1)$ & $23(1)$ \\
\hline$C(19)$ & $63(1)$ & $55(1)$ & $46(1)$ & $12(1)$ & $10(1)$ & $8(1)$ \\
\hline$C(20)$ & $32(1)$ & $43(1)$ & $48(1)$ & $0(1)$ & $2(1)$ & $-1(1)$ \\
\hline$C(21)$ & $68(1)$ & $44(1)$ & $40(1)$ & $8(1)$ & $19(1)$ & $16(1)$ \\
\hline$C(22)$ & $139(3)$ & $52(2)$ & $64(2)$ & $-15(1)$ & $4(2)$ & $29(2)$ \\
\hline$O(51)$ & $55(1)$ & $48(1)$ & $51(1)$ & $2(1)$ & $21(1)$ & $14(1)$ \\
\hline$O(52)$ & $34(1)$ & $37(1)$ & $38(1)$ & $4(1)$ & $3(1)$ & $4(1)$ \\
\hline$O(53)$ & $85(1)$ & $49(1)$ & $60(1)$ & $12(1)$ & $1(1)$ & $-21(1)$ \\
\hline$O(54)$ & $59(1)$ & $51(1)$ & $56(1)$ & $0(1)$ & $12(1)$ & $19(1)$ \\
\hline O (55) & $39(1)$ & $68(1)$ & $45(1)$ & $-5(1)$ & $6(1)$ & $9(1)$ \\
\hline$O(56)$ & $58(1)$ & $52(1)$ & $29(1)$ & $-2(1)$ & $-2(1)$ & $1(1)$ \\
\hline$O(57)$ & $63(1)$ & $87(1)$ & $57(1)$ & $22(1)$ & $-19(1)$ & $-4(1)$ \\
\hline$O(58)$ & $77(1)$ & $44(1)$ & $61(1)$ & $1(1)$ & $13(1)$ & $-4(1)$ \\
\hline$O(59)$ & $77(1)$ & $46(1)$ & $56(1)$ & $-9(1)$ & $27(1)$ & $-15(1)$ \\
\hline C (51) & $37(1)$ & $40(1)$ & $35(1)$ & $2(1)$ & $7(1)$ & $0(1)$ \\
\hline C (52) & $45(1)$ & $38(1)$ & $40(1)$ & $3(1)$ & $9(1)$ & $3(1)$ \\
\hline C (53) & $38(1)$ & $40(1)$ & 35 (1) & $1(1)$ & $8(1)$ & $3(1)$ \\
\hline C (54) & $37(1)$ & $48(1)$ & $30(1)$ & $-2(1)$ & $7(1)$ & $2(1)$ \\
\hline C (55) & $32(1)$ & $46(1)$ & $29(1)$ & $3(1)$ & $6(1)$ & $6(1)$ \\
\hline$C(56)$ & $32(1)$ & $37(1)$ & $29(1)$ & $4(1)$ & $5(1)$ & $6(1)$ \\
\hline C (57) & $37(1)$ & $38(1)$ & $36(1)$ & $4(1)$ & $4(1)$ & $2(1)$ \\
\hline C (58) & $55(1)$ & $39(1)$ & $37(1)$ & $6(1)$ & $9(1)$ & $2(1)$ \\
\hline$C(59)$ & $84(2)$ & $53(1)$ & $47(1)$ & $15(1)$ & $16(1)$ & $27(1)$ \\
\hline$C(60)$ & $64(1)$ & $40(1)$ & $44(1)$ & $1(1)$ & $12(1)$ & $8(1)$ \\
\hline$C(61)$ & $48(1)$ & $42(1)$ & $33(1)$ & $2(1)$ & $1(1)$ & $-3(1)$ \\
\hline$C(62)$ & $36(1)$ & $42(1)$ & $27(1)$ & $0(1)$ & $2(1)$ & $2(1)$ \\
\hline$C(63)$ & $40(1)$ & $45(1)$ & $26(1)$ & $3(1)$ & $2(1)$ & $2(1)$ \\
\hline$C(64)$ & $50(1)$ & $46(1)$ & $31(1)$ & $3(1)$ & $10(1)$ & $-3(1)$ \\
\hline$C(65)$ & $39(1)$ & $47(1)$ & $48(1)$ & $7(1)$ & $5(1)$ & $0(1)$ \\
\hline$C(66)$ & $50(1)$ & $49(1)$ & $49(1)$ & $-3(1)$ & $8(1)$ & $-10(1)$ \\
\hline$C(67)$ & $56(1)$ & $58(1)$ & $84(2)$ & $4(1)$ & $29(1)$ & $-6(1)$ \\
\hline$C(68)$ & $50(1)$ & $59(1)$ & $44(1)$ & $-13(1)$ & $-1(1)$ & $0(1)$ \\
\hline C (69) & $63(1)$ & $52(1)$ & $37(1)$ & $6(1)$ & $13(1)$ & $0(1)$ \\
\hline$C(70)$ & $45(1)$ & $50(1)$ & $25(1)$ & $-3(1)$ & $4(1)$ & $12(1)$ \\
\hline$C(71)$ & $63(1)$ & $77(2)$ & $33(1)$ & $5(1)$ & $-7(1)$ & $-13(1)$ \\
\hline$C(72)$ & $174(4)$ & $124(3)$ & $42(1)$ & $-25(2)$ & $-14(2)$ & $-5(3)$ \\
\hline$O(91)$ & $104(2)$ & $178(3)$ & $52(1)$ & $-38(1)$ & $33(1)$ & $-92(2)$ \\
\hline O (92) & $67(6)$ & $79(7)$ & $122(9)$ & $60(7)$ & $-3(6)$ & $-2(5)$ \\
\hline
\end{tabular}

$\& E$

\& J3

$\& F$

\&W6 64

\&! Table 4. Hydrogen coordinates ( $\mathrm{x} 10 \& \wedge 4 \& 0)$ and isotropic 
displacement parameters $\left(\& G A \&^{\wedge} 2 \& 0 \times 10 \&^{\wedge} 3 \& 0\right)$ for ar035.\&H $\& \mathrm{C}$

\begin{tabular}{lllll}
\hline$\& E$ & & & & \\
$\& J 2$ & & $y$ & & \\
$\& C$ & $x$ & $y$ & & \\
& & & & \\
\end{tabular}

$\& \mathrm{E}$

$\& \mathrm{C}$

$\mathrm{H}(1)$

$\mathrm{H}(3)$

9201

$\mathrm{H}(8)$

8451

$\mathrm{H}(1 \mathrm{~A})$

7442

$\mathrm{H}(2)$

5740

H (5)

8131

$\mathrm{H}(7)$

5608

$\mathrm{H}(9 \mathrm{~A})$

8380

$\mathrm{H}(9 \mathrm{~B})$

5213

$\mathrm{H}$ (10)

5611

$\mathrm{H}$ (11)

5718

$\mathrm{H}(13)$

7391

$\mathrm{H}$ (14A)

7858

$\mathrm{H}$ (14B)

7047

$\mathrm{H}(16)$

5746

$\mathrm{H}(17 \mathrm{~A})$

6018

4763

$\mathrm{H}(17 \mathrm{~B})$

4595

$\mathrm{H}(18 \mathrm{~A})$

5488

$\mathrm{H}(18 \mathrm{~B})$

6790

$\mathrm{H}(18 \mathrm{C})$

5993

$\mathrm{H}(19 \mathrm{~A})$

6824

7962

$\mathrm{H}(19 \mathrm{C}) \quad 6693$

$\mathrm{H}(22 \mathrm{~A}) \quad 7951$

$\mathrm{H}(22 \mathrm{~B}) \quad 7212$

$\mathrm{H}(22 \mathrm{C}) \quad 6591$

$\mathrm{H}(51)$

175

$\mathrm{H}(53)$

$\mathrm{H}(58)$

$\mathrm{H}(51 \mathrm{~A})$

$-1361$

2858

2986

$\mathrm{H}(52) \quad 1007$

$\mathrm{H}(55)$

$\mathrm{H}(57)$

$\mathrm{H}(59 \mathrm{~A})$

2401

$-698$

2003

$\mathrm{H}(59 \mathrm{~B})$

1201

$\mathrm{H}(60)$

$\mathrm{H}(61)$

$\mathrm{H}(63)$

$\mathrm{H}(64 \mathrm{~A})$

$\mathrm{H}(64 \mathrm{~B})$

$\mathrm{H}(66)$

$\mathrm{H}(67 \mathrm{~A})$

$\mathrm{H}(67 \mathrm{~B})$

$\mathrm{H}(68 \mathrm{~A})$

1283

95

470

1749

2839

3892

4681

4339

$\mathrm{H}(68 \mathrm{~B})$

3385

$\mathrm{H}(68 \mathrm{C})$

3061

2288

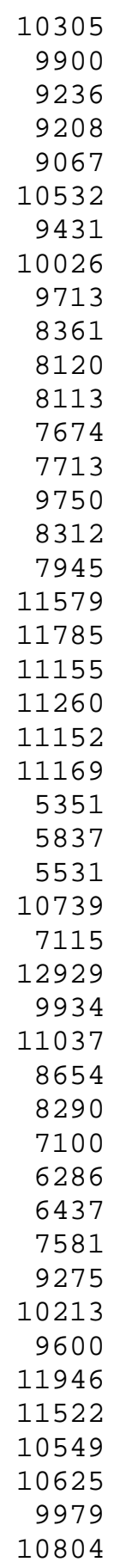

$\begin{array}{rr}11337 & 68 \\ 6083 & 74 \\ 14521 & 104\end{array}$

$10875 \quad 47$

$11730 \quad 47$

$8872 \quad 44$

$7870 \quad 41$

$6883 \quad 58$

$5757 \quad 58$

$6310 \quad 55$

$7514 \quad 44$

$9757 \quad 44$

$11273 \quad 53$

$10732 \quad 53$

$13597 \quad 67$

$13437 \quad 90$

$12218 \quad 90$

$10489 \quad 92$

$10962 \quad 92$

$11577 \quad 92$

$6299 \quad 81$

$7102 \quad 81$

$7546 \quad 81$

$9031 \quad 128$

$8096 \quad 128$

$9120 \quad 128$

$7515 \quad 76$

$6456 \quad 97$

$5089 \quad 90$

$5473 \quad 44$

$5018 \quad 49$

$7421 \quad 43$

$5953 \quad 44$

$7197 \quad 73$

$6958 \quad 73$

$5170 \quad 58$

$4483 \quad 49$

$4025 \quad 44$

$3427 \quad 50$

$3621 \quad 50$

$5850 \quad 59$

$3883 \quad 78$

$3564 \quad 78$

$\begin{array}{ll}7706 & 77\end{array}$

$8606 \quad 77$

$8381 \quad 77$ 
S17

$\begin{array}{lrrrr}\text { H }(69 A) & 237 & 7227 & 8644 & 75 \\ \text { H }(69 B) & -489 & 8042 & 8260 & 75 \\ \text { H (69C) } & 909 & 8058 & 8330 & 75 \\ \text { H (72A) } & 1868 & 8116 & 1116 & 172 \\ \text { H }(72 B) & 543 & 8249 & 655 & 172 \\ \text { H }(72 C) & 853 & 7544 & 1532 & 172 \\ \text { H }(101) & 9376 & 382 & 9255 & 165 \\ \text { H }(201) & 10269 & 977 & 9296 & 165\end{array}$

